\title{
ON TWO EXTREMUM PROBLEMS RELATED TO THE NORM OF A BOUNDED LINEAR OPERATOR
}

\author{
Debmalya Sain, Kallol Paul and Kalidas Mandal
}

\begin{abstract}
We explore the norm attainment set and the minimum norm attainment set of a bounded linear operator between Hilbert spaces and Banach spaces. Indeed, we obtain a complete characterization of both the sets, separately for operators between Hilbert spaces and Banach spaces. We also study the interconnection between these two sets and prove that for operators between Hilbert spaces, these two sets are either equal or mutually orthogonal, provided both of them are non-empty. We also obtain separate complete characterizations of reflexive Banach spaces and Euclidean spaces in terms of the norm (minimum norm) attainment set, in order to illustrate the importance of our study.
\end{abstract}

Mathematics subject classification (2010): 47L05, 46B20, 46C15.

Keywords and phrases: Orthogonality, linear operators, norm attainment.

\section{REFERENCES}

[1] BiRKhoff, G., Orthogonality in linear metric spaces, Duke Math. J., 1 (1935), 169-172.

[2] Carvajal, X. And Neves, W., Operators that achieve the norm, Integral Equations Operator Theory, 72 (2012), 179-195.

[3] Carvajal, X. and Neves, W., Operators that attain their minima, Bull. Braz. Math. Soc., (N. S.) 45(2) (2014), 293-312.

[4] Giles, J. R., Classes of semi-inner-product spaces, Trans. Amer. Math. Soc., 129 (1967), 436-446.

[5] James, R. C., Inner products in normed linear spaces, Bull. Amer. Math. Soc., 53(6) (1947), 559566.

[6] James, R. C., Orthogonality and linear functionals in normed linear spaces, Trans. Amer. Math. Soc., 61 (1947), 265-292.

[7] JAMES, R. C., Reflexivity and the sup of linear functionals, Israel J. Math., 13 (1972), 289-300(1973).

[8] Lumer, G., Semi-inner-product spaces, Trans. Amer. Math. Soc., 100 (1961), 29-43.

[9] Paul, K., Sain, D., Mal, A. And Mandal, K., Orthogonality of bounded linear operators on complex Banach spaces, Adv. Oper. Theory 3 (2018), no. 3, 699-709.

[10] SAIN, D., Birkhoff-James orthogonality of linear operators on finite dimensional Banach spaces, J. Math. Anal. Appl., 447 (2017), 860-866.

[11] SAIN, D., On the norm attainment set of a bounded linear operator, J. Math. Anal. Appl., 457 (2018), $67-76$.

[12] SAIN, D., On extreme contractions and the norm attainment set of a bounded linear operator, Ann. Funct. Anal. 10 (2019), no. 1, 135-143.

[13] SAIN, D., On the norm attainment set of a bounded linear operator and semi-inner-products in normed spaces, arXiv:1802.10439v2 [math.FA], Indian J. Pure Appl. Math., Accepted.

[14] SAIN, D. AND PAUL, K., Operator norm attainment and inner product spaces, Linear Algebra Appl., 439 (2013), 2448-2452. 